\title{
Effect of the Jupiter magnetosphere on the cosmic ray protons measured with the PAMELA experiment
}

\author{
M. Ricci ${ }^{* 20}$, G. Pizzella ${ }^{20}$, M. Martucci ${ }^{11,20}$, A. Bruno ${ }^{6}$, V. Di Felice ${ }^{10,19}$, N. Marcelli ${ }^{11}$, O. Adriani ${ }^{1,2}$, G. C. \\ Barbarino $^{3,4}$, G. A. Bazilevskaya ${ }^{5}$, R. Bellotti ${ }^{6,7}$, M. Boezio $^{8}$, E. A. Bogomolov ${ }^{9}$, M. Bongi ${ }^{1,2}$, V. \\ Bonvicini $^{8}$, F. Cafagna ${ }^{7}$, D. Campana ${ }^{4}$, P. Carlson ${ }^{13}$, M. Casolino ${ }^{10,14}$, G. Castellini ${ }^{15}$, C. De Santis ${ }^{10}$, A. \\ M. Galper ${ }^{12}$, A. V. Karelin ${ }^{12}$, S. V. Koldashov ${ }^{12}$, S. Koldobskiy ${ }^{12}$, S. Y. Krutkov ${ }^{9}$, A. N. Kvashnin ${ }^{5}$, A. \\ Leonov $^{12}$, V. Malakhov ${ }^{12}$, L. Marcelli ${ }^{10}$, A. G. Mayorov ${ }^{12}$, W. Menn ${ }^{17}$, M. Mergè ${ }^{10,11}$, V. V. Mikhailov ${ }^{12}$, E. \\ Mocchiutti $^{8}$, A. Monaco ${ }^{6,7}$, N. Mori ${ }^{2}$, R. Munini ${ }^{8,16}$, G. Osteria ${ }^{4}$, B.Panico ${ }^{4}$, P. Papini ${ }^{2}$, M. Pearce ${ }^{13}$, P. \\ Picozza $^{10,11}$, S. B. Ricciarini ${ }^{2,15}$, M. Simon ${ }^{17}$, R. Sparvoli ${ }^{10,11}$, P. Spillantini ${ }^{2,15}$, Y. I. Stozhkov ${ }^{5}$, A. \\ Vacchi $^{8,22}$, E. Vannuccini ${ }^{2}$, G. Vasilyev ${ }^{9}$, S. A. Voronov ${ }^{12}$, Y. T. Yurkin ${ }^{12}$, G. Zampa ${ }^{8}$ and N. Zampa ${ }^{8}$. \\ ${ }^{1}$ University of Florence, Department of Physics, I-50019 Sesto Fiorentino, Florence, Italy; ${ }^{2}$ INFN, Sezione di Florence, \\ I-50019 Sesto Fiorentino, Florence, Italy, ${ }^{3}$ University of Naples "Federico II", Department of Physics, I-80126 Naples, \\ Italy; ${ }^{4}$ INFN, Sezione di Naples, I-80126 Naples, Italy; ${ }^{5}$ Lebedev Physical Institute, RU-119991, Moscow, Russia; \\ ${ }^{6}$ University of Bari, Department of Physics, I-70126 Bari, Italy; ${ }^{7}$ INFN, Sezione di Bari, I-70126 Bari, Italy; ${ }^{8}$ INFN, \\ Sezione di Trieste, I-34149 Trieste, Italy; ${ }^{9}$ Ioffe Physical Technical Institute, RU-194021 St. Petersburg, Russia; ${ }^{10}$ INFN, \\ Sezione di Rome "Tor Vergata", I-00133 Rome, Italy; ${ }^{11}$ University of Rome "Tor Vergata", Department of Physics, \\ I-00133 Rome, Italy; ${ }^{12}$ MEPhI: National Research Nuclear University MEPhI, RU-115409, Moscow, Russia; ${ }^{13} \mathrm{KTH}$, \\ Department of Physics, and the Oskar Klein Centre for Cosmoparticle Physics,; AlbaNova University Centre, SE-10691 \\ Stockholm, Sweden; ${ }^{14}$ RIKEN, EUSO team Global Research Cluster, Wako-shi, Saitama, Japan; ${ }^{15}$ IFAC, I-50019 Sesto \\ Fiorentino, Florence, Italy; ${ }^{16}$ University of Trieste, Department of Physics, I-34147 Trieste, Italy; ${ }^{17}$ Universitat Siegen, \\ Department of Physics, D-57068 Siegen, Germany; ${ }^{18}$ INFN, Sezione di Perugia, I-06123 Perugia, Italy; ${ }^{19}$ Agenzia \\ Spaziale Italiana (ASI) Science Data Center, Via del Politecnico snc, I-00133 Roma, Italy, ${ }^{20}$ INFN, Laboratori Nazionali \\ di Frascati, Via Enrico Fermi 40, I-00044 Frascati, Italy; ${ }^{21}$ Istituto Nazionale di Astrofisica, Fosso del cavaliere 100, \\ 00133 Roma, Italy; ${ }^{22}$ University of Udine, Department of Mathematics, Computer Science and Physics Via delle Scienze, \\ 206, Udine, Italy.
}

\begin{abstract}
The very intense Jovian magnetic field produces a magnetosphere where high-energy charged particles are trapped, allowing the possibility for acceleration mechanism that could inject those particles in the open space. In the last decades, accelerated electrons from the Jupiter magnetosphere have been detected and studied in the interplanetary space. This work investigated whether the proton data obtained by the PAMELA space-borne detector between July $9^{\text {th }}$, 2006 to August $31^{\text {th }}, 2014$ shows signatures that arise from Jupiter. In this proceeding the basis of the analysis of cosmic ray protons by PAMELA are described and results will be shown at the conference.
\end{abstract}

35th International Cosmic Ray Conference - ICRC2017-

10-20 July, 2017

Bexco, Busan, Korea

\footnotetext{
* Speaker.
} 


\section{Introduction}

It is well known that Jupiter has a very high magnetic field moment $M_{J}=1.56 \times 10^{20} \mathrm{Tm}^{3}$, $\sim 10^{4}$ larger than the one of Earth, which induces an intense magnetosphere. This intensity, together with the general weakness of the solar wind at Jupiter, forms the ideal conditions to generates a powerful magnetosphere. Strong evidence of acceleration mechanism in magnetospheric environments has been found on Earth, where trapped electrons are accelerated to relativistic energies by local mechanism inside the Van Allen radiations belts [1]. For this reason, it is natural to ask whether the Jupiter magnetosphere could also be capable of activating similar acceleration mechanism too. Evidences of high energy electrons accelerated by Jupiter have been found (see [2], but not for protons [3] The fact that some cosmic rays observed on Earth could be accelerated in the Jupiter magnetosphere, at least up to energies of the order of few $\mathrm{GeV}$, has been discussed in the past using observations made by ground stations [4]. To better understand the mechanisms of transport in the inner part of the heliosphere (up to $5 \mathrm{AU}$, where Jupiter is located) a study of the Interplanetary Magnetic Field (IMF) must be carried out. The IMF plays a crucial role in particle transport in the solar system due to its spiral field lines, described classically by the Parker theory [5] It can be shown from the equation of the IMF lines in the ecliptic plane, assuming the Parker classic model, that the relative angle for the Earth-Jupiter system in which they are magnetically connected corresponds to $\Phi_{E J}=216^{\circ}$. All the past and present experiments with space-borne detectors give an IMF with spiral field lines, but the precise behavior depends on many external factors. For example, data obtained by the Pioneer 11, Voyager and Ulysses spacecraft show some discrepancies from the classical Parker theory and it turns out that the angle $\Phi_{E J}$ can vary between $95^{\circ}$ and $140^{\circ}$ [6]. Data of cosmic ray protons obtained by neutron monitors yield a value of $\sim 125^{\circ}$, while the work described in [7] instead gives $\Phi_{E J} \sim 220^{\circ}$. A scheme of the configuration Earth-Sun-Jupiter is shown in Figure 1, with the angle $\Phi_{E J}$ between the three astrophysical objects.

\section{The PAMELA mission: overview}

The PAMELA (a Payload for Antimatter-Matter Exploration and Light-nuclei Astro- physics) satellite experiment is designed to study the charged component of the cosmic radiation, focusing on antiparticles. PAMELA was launched with a Soyuz-U rocket on June 15th of 2006 from the Baikonur cosmodrome (Kazakhstan). The apparatus is hosted on the Russian Resurs-DK1 satellite, a commercial Earth-observation spacecraft. At first the orbit was elliptical (altitude varying between 355 and $584 \mathrm{~km}$ ) and semipolar (inclination of about 70 ${ }^{\circ}$ ) and with a period of about 94 minutes, see Figure 2 (left). In 2010 the orbit was set to be circular with an almost fixed altitude of about $580 \mathrm{~km}$. The mission was planned to last 3 years, but take data for almost 10 years. This experiment could be considered as the greatest effort of the WiZARD Collaboration which, starting almost 30 years ago with the New Mexico State University group led by the late Robert L. Golden (July $28^{\text {th }} 1940$ - April $7^{\text {th }}$ 1995), has successfully built and own several balloon-borne experiments like MASS-89, MASS-91, Tramp-Si93, CAPRICE-94 and CAPRICE-98 and also satellites like NINA-1 (1998) and NINA-2 (2000). The WiZARD group has also pioneered some innovative detection techniques such as electromagnetic calorimeters, superconducting magnet spectrometers and ring imaging Cerenkov detectors in balloon flights. PAMELA has greatly contributed to the 


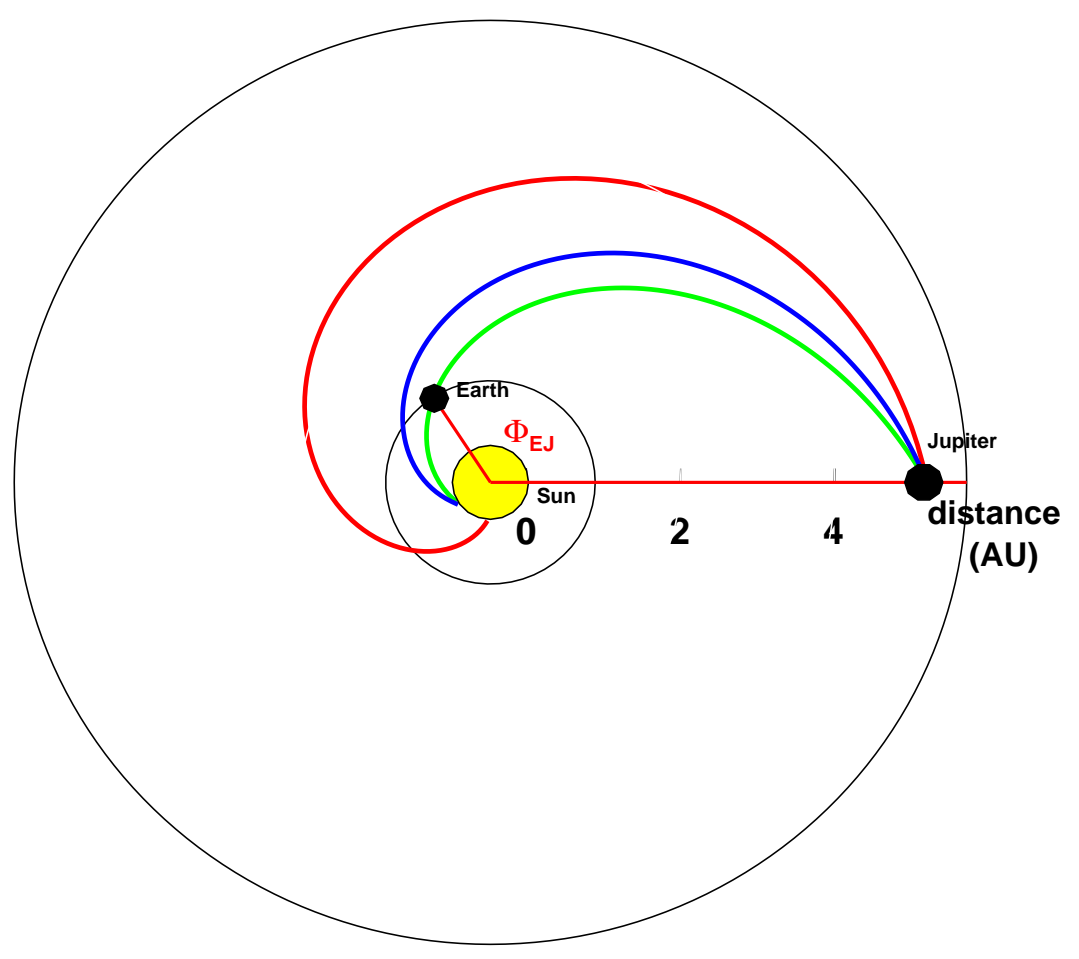

Figure 1: A schematic view of the Earth-Sun-Jupiter system with the magnetic field lines connecting Jupiter with Earth. The green line refers to $\gamma=0.37\left(\Phi_{E J}=117^{\circ}\right)$, the blue line to $\gamma=0.57\left(\Phi_{E J}=140^{\circ}\right)$ and the red line to $\gamma=1\left(\Phi_{E J}=216^{\circ}\right)$. The angle $\Phi_{E J}$ describes the geometrical angle between Earth and Jupiter.

advancement of knowledge in many fields of astrophysics, measuring the antiproton spectrum up to $100 \mathrm{GeV}$, the positron spectrum up to $200 \mathrm{GeV}$, the electron spectrum up to $600 \mathrm{GeV}$, Boron and Carbon nuclei spectra (1-200 GV) and mostly it gave the first high-precision measurements of the proton and helium nuclei spectra up to 1.2 TV. A comprehensive review of the results obtained by PAMELA can be found in [8]

\section{The detector}

The PAMELA detector was equipped with various instruments which guaranteed a great redundancy in case of unexpected malfunctioning of some sub-detectors and allowed to perform in-flight calibrations, evaluate efficiencies and provide different measurements of a single event. The detector is composed of the following systems:

- a Time-of-Flight (ToF) system, with segmented scintillator planes divided into 3 groups $\left(S_{1}\right.$, $\left.S_{2}, S_{3}\right)$.

- a magnetic spectrometer to measure trajectories of the incoming particles 
- an anticoincidence system with solid scintillators (CARD, CAT, CAS)

- an electromagnetic sampling calorimeter

- a shower tail-catcher scintillator

- a neutron detector

In Figure 2 (right) a sketch of the isometric view of the whole detector with all the sub-detetctors is shown.
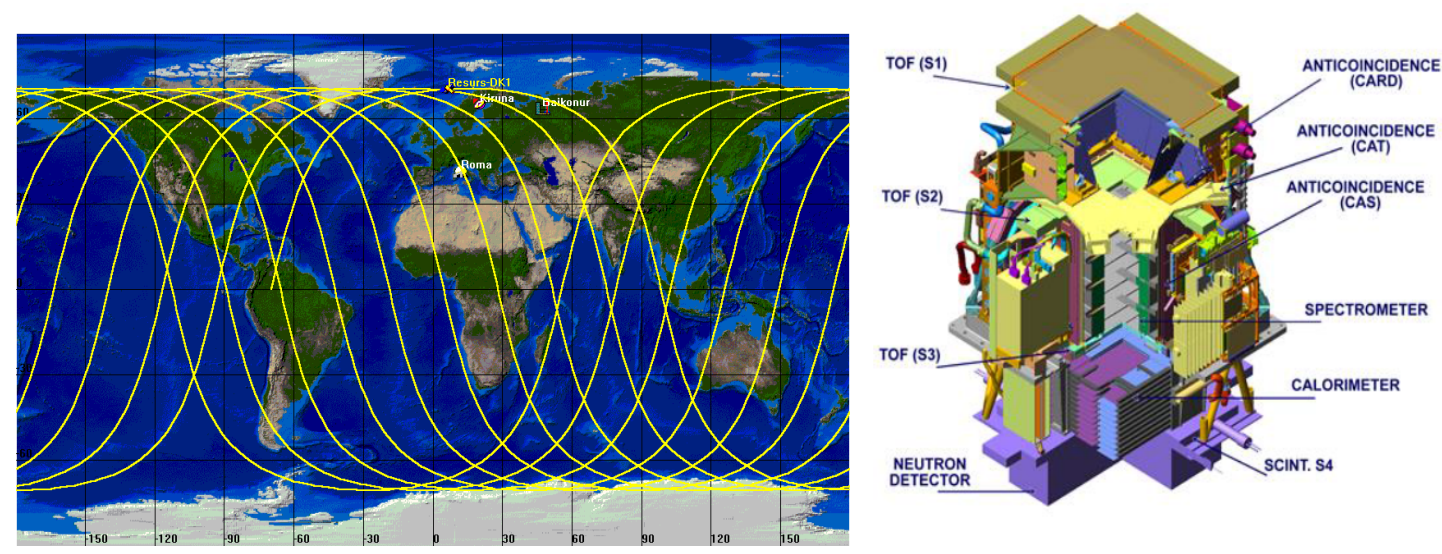

Figure 2: Orbit of PAMELA (left) and a scheme of the detector (right) with all the sub-detectors.

The core of the instrument is a Nd-Fe-B alloy permanent magnet (with residual magnetization of $1.3 \mathrm{~T}$ ) with a microstrip silicon tracking system composed of 6 equidistant $300 \mu \mathrm{m}$ thick planes that constitute a magnetic spectrometer used to determine the rigidity $(R=p c / Z e)$ and the charge $Z$ of particles crossing the cavity. The double-sided silicon sensors provide two independent impact coordinates on each plane and the blocks of the magnet are configured to provide an almost uniform magnetic field oriented along the $y$-direction inside a cavity $\left(13.1 \times 16.1 \mathrm{~cm}^{2}\right)$. The dimensions of the permanent magnet define the maximum geometrical factor of the PAMELA experiment to be $21.6 \mathrm{~cm}^{2}$ sr. A set of scintillators forms the anticoincidence system, which consists of 4 plastic scintillators (CAS) surrounding the sides of the magnet, one covering the top (CAT) and a set of 4 plastic scintillators (CARD) that surrounds the volume between the first two Time-of-Flight planes. An electromagnetic imaging calorimeter, placed under the spectrometer, comprises 44 single-sided silicon sensor layers ( $380 \mu \mathrm{m}$ thick) interleaved with 22 plates of tungsten absorber. It can distinguish between leptons and hadrons through the analysis of the shape of the shower they produce. It also measures the energy of electrons and positrons interacting in its volume and check the alignment of the tracker sensors. A Time-of-Flight system, composed of 6 layers of fast plastic scintillators arranged in 3 double planes $\left(S_{1}, S_{2}\right.$ and $\left.S_{3}\right)$ provides information on particle velocity $\beta$ and trigger. More details on the instrument can be found in Ref [8]. 


\section{The analysis: calculation of the proton flux}

\section{Particle identification in the instrument}

Since energy loss of a charged particle through matter follows the Bethe Bloch formula, (neglecting the logarithmic term), the measurement of the average energy released in the tracker planes at a given rigidity can be used to discriminate between different particle species. Proton candidates are selected requiring energy loss in the tracker planes compatible with $\mathrm{Z}=1$ (MIP in most of the PAMELA rigidity range). These selections in the energy loss as a function of rigidity $\mathrm{R}$ remove positrons, pions and particles with $\mathrm{Z}>1$. This can be seen in Figure 3 At low rigidities, background pions and other secondary particles are clearly distinguishable. In this work, no selection to get rid of hydrogen isotopes has been applied.

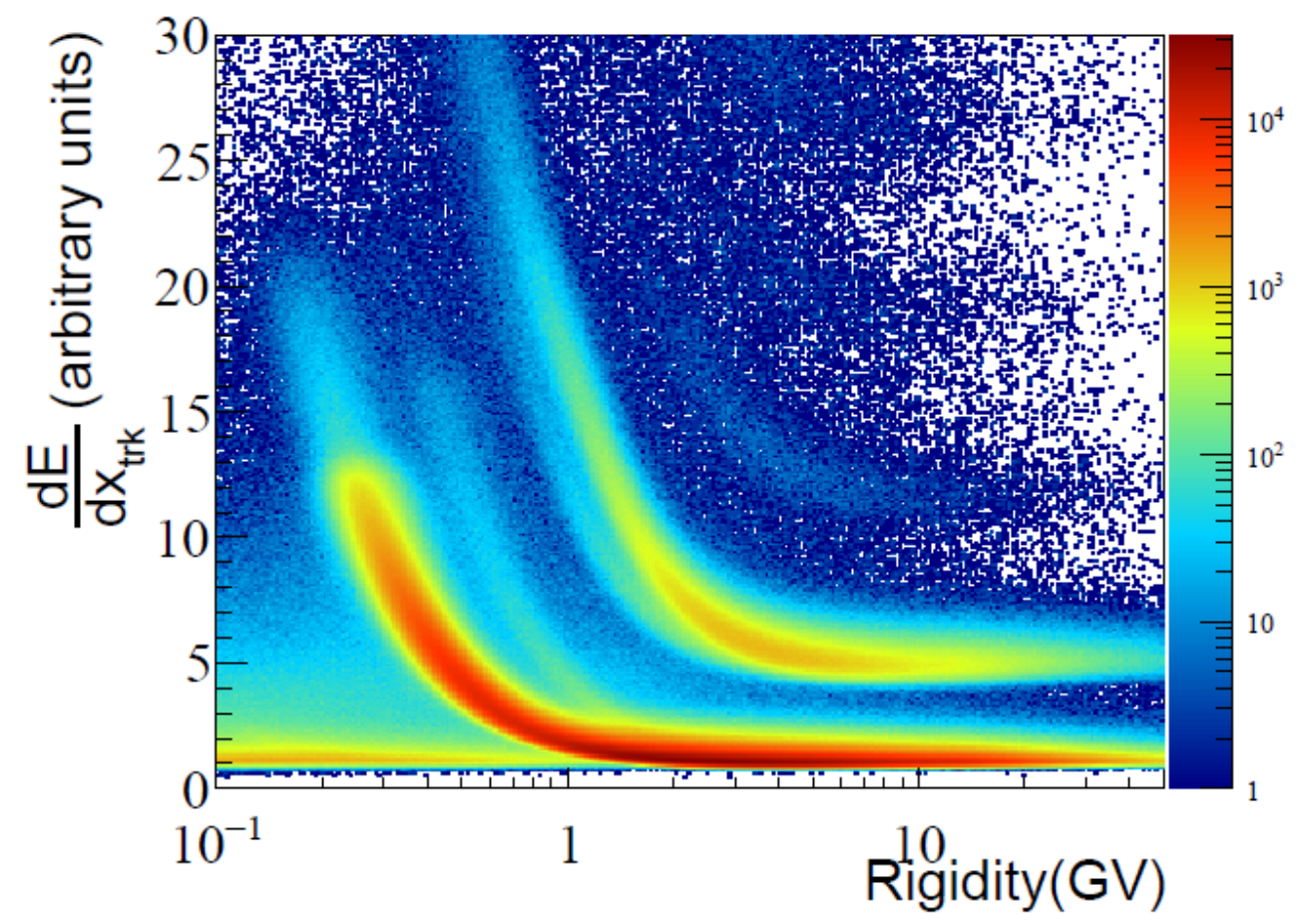

Figure 3: Energy released in the six planes of the tracker as a function of rigidity. The band of Hydrogen and Helium nuclei are clearly distinguishable and can be easily separated. Secondary particles in the bottom left part of the plot are excluded.

\section{Geometrical factor}

The geometrical factor (GF) of PAMELA was evaluated by defining a fiducial area of $0.15 \mathrm{~cm}$ from the internal walls of the magnetic cavity. Only particles passing through this fiducial volume are used in the analysis and become part of the flux sample. The definition of this kind of ideal structure ensures that all particles entering the cavity itself can cross all the scintillators without hitting the magnet itself. The value of GF $=14.55 \mathrm{vcm}^{2} \mathrm{sr}$, estimated with a numerical calculation and cross-checked with the simulation is found to be constant above $1 \mathrm{GV}$ within $0.1 \%$. 


\section{Live Time and geomagnetic cutoff}

The live time of the apparatus are evaluated using information from the trigger system. Counters for both live and dead time are cross-checked with the on-board time of the CPU; the total errors on live time measurements are negligible compared to other sources of uncertainties. In this analysis we are interested in isolating galactic protons from other populations of protons (reentrant, precipitating, etc.) so a selection is developed to avoid any contamination. This selection is carried out using the magnetic field of the Earth and its configuration; in the polar region the geomagnetic cutoff rigidity ${ }^{1}$ is minimal while in the equatorial regions it is maximum. Therefore, the protons collected at the poles will be a galactic, while that measured near the equator will be a galactic only above a certain geomagnetic rigidity while below the contamination of reentrant, albedo and other sub-cutoff particles becomes greater. The selection $\mathrm{R}_{\text {measured }}>1.3 \times \mathrm{R}_{\text {cutoff }}$ ensures the rejection of all these contaminating populations.

\section{Solar Energetic Particles rejection}

During the 9 years of data taking, analyzed in this work, PAMELA registered almost 29 solar events. These inject a huge number of very energetic particles, called SEP (Solar Energetic Particles), in the interplanetary space. For this reason, the low energy galactic particles in the heliosphere are often mixed to particles of solar origin, so their contribution must be excluded from data. A careful search of these events and their effect in PAMELA data has been carried out and the days during which these solar events took place are cut out from the data-set.

\section{Periodicity and angular distributions}

We analyze the proton data obtained with PAMELA in the period July 2006 -August 2014, taking daily averages as a function of rigidity. A typical example of measured proton distribution is shown in Figure 4, up to $15 \mathrm{GV}$.

The proton daily fluxes as a function of time is then evaluated in two rigidity intervals, with the idea to verify whether this behavior shows some signature due to the presence of Jupiter magnetosphere. A total of $\sim 2600$ daily proton intensities have been collected; the whole data-set includes a period in which solar activity is minimum (2006-2009) and the subsequent maximum of the $24^{\text {th }}$ solar cycle (the flux decreases due to an increased turbulence in the heliosphere). To get rid of the solar modulation effect, a fit with a simple third-degree polynomial is carried out and then this effect is subtracted to the data to highlight any variations with higher frequency. Studying the periodicity of these variations, if present, is a good way to understand their origin. Moreover, we study the spatial distribution of the measured proton to search for a possible privileged arrival direction from the sector of the heliosphere where Jupiter is located. Knowing the relative position between Earth, Sun and Jupiter on a daily basis, and knowing their relative angle $\Phi_{E J}$ it is possible to investigate if the number of galactic protons rises for specific values of this angle.

\footnotetext{
${ }^{1}$ defined as the minimum rigidity necessary for a galactic charged particle, ideally approaching from infinity, to reach that point on the Earth magnetosphere
} 


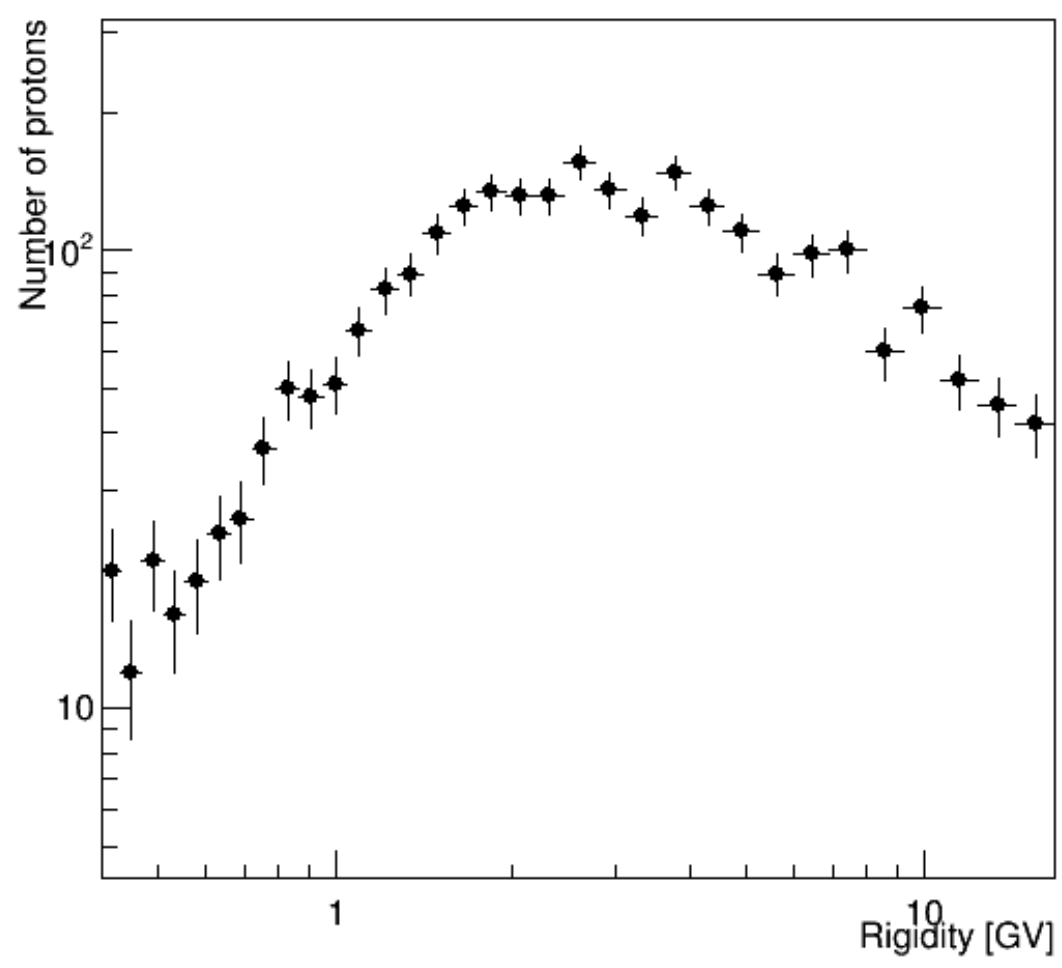

Figure 4: A typical proton distribution measured by PAMELA as a function of rigidity, averaged over one day.

\section{Results and conclusions}

A higher contribution of protons is registered when Earth is orbiting around the Sun with angles $\Phi_{E J}$ in the range, roughly, $60^{\circ}-150^{\circ}$; these angles are in reasonable agreement with those where the measured IMF lines intersect the Earth orbit. Thus, more protons are detected at Earth in the region where the IMF lines connect Jupiter with Earth. Also, the intensity profile of the fluxes contains a periodic component in good agreement with the synodic period of Jupiter $(\sim 398.88$ days). Results will be presented as a poster in this conference.

\section{Acknowledgements}

The Italian authors acknowledge the partial financial support from the Italian Space Agency (ASI) under the program "Programma PAMELA - attività scientifica di analisi dati in fase E". We also acknowledge support from Deutsches Zentrum für Luft-und Raumfahrt (DLR), The Swedish National Space Board, The Swedish Research Council, The Russian Space Agency (Roscosmos), and Russian Science Foundation (Grant No. 14-12-00373). M. P. and E. E. V. acknowledge partial financial support from the the South African National Research Foundation (NRF) under their Research Cooperation Programme. 


\section{References}

[1] G. D. Reeves, et al., Science 341, 991 (2013).

[2] B. J. Teegarden, F. B. McDonald, J. H. Trainor, W. R. Webber, E. C. Roelof, JGR 79, 3615 (1974).

[3] J. A. Simpson, et al., Science 183, 306 (1974)

[4] G. Pizzella, Nature 226, 434 (1970).

[5] E. Smith, A. Balogh, M. Burton, R. Forsyth, R. Lepping, Advances in Space Research 20, 47 (1997).

[6] G. Pizzella, Nuovo Cimento Lettere 13, 179 (1975).

[7] B. Mitra, S. K. Bose, S. R. Ganguly, International Cosmic Ray Conference 3, 182 (1983).

[8] O. Adriani, et al., Phys. Rep. 544, 323 (2014). 